\title{
Low-Dose Valganciclovir for CMV Prophylaxis after Lung Transplantation
}

\author{
Hargobind S. Khurana, ${ }^{1}$ Alison Kole, ${ }^{1}$ Jeremy Falk, ${ }^{1}$ Sara Ghandehari, ${ }^{1}$ Guy Soohoo, ${ }^{2}$ \\ Sinan Simsir, ${ }^{3}$ Wen Cheng, ${ }^{3}$ Robert M. Kass, ${ }^{3}$ Jasjit Khurana, ${ }^{1}$ and George Chaux ${ }^{1}$ \\ ${ }^{1}$ Pulmonary and Critical Care, Cedars-Sinai Medical Center, Los Angeles, CA 90048, USA \\ ${ }^{2}$ Pulmonary and Critical Care, West Los Angeles VA Healthcare Center, Los Angeles, CA 90073, USA \\ ${ }^{3}$ Cardio-Thoracic Surgery, Cedars-Sinai Medical Center, Los Angeles, CA 90048, USA \\ Correspondence should be addressed to Hargobind S. Khurana; hskhurana@yahoo.com
}

Received 21 October 2012; Accepted 6 November 2012

Academic Editors: W. Lim and H. Toz

Copyright (C) 2013 Hargobind S. Khurana et al. This is an open access article distributed under the Creative Commons Attribution License, which permits unrestricted use, distribution, and reproduction in any medium, provided the original work is properly cited.

\begin{abstract}
Purpose. Cytomegalovirus (CMV) remains an important pathogen following solid organ transplantation (SOT). Universal prophylaxis for CMV is adopted by most centers after lung transplantation. Various combinations studied for CMV prophylaxis include intravenous and oral ganciclovirs, oral valganciclovir, and CMV immunoglobulins. We present our experience with a low-dose of oral valganciclovir for CMV prophylaxis following lung transplantation. Methods and Materials. Our center started using $450 \mathrm{mg}$ of daily oral valganciclovir for CMV prophylaxis in lung transplant recipients in Jan, 2001. A retrospective chart analysis of patients who underwent lung transplantation from January 2001 to December 2006 was done. Of 46 patients, 4 were excluded as they died within 30 days of transplant from postop complications. The mean age at transplant was 64 years, mostly single lung transplants (36/6) with a male-to-female ratio of 25/17. COPD was the most common reason for transplant (65\%), and the serological CMV status of donors (D) and recipients (R) was as follows: $\mathrm{D}+/ \mathrm{R}+28, \mathrm{D}+/ \mathrm{R}-5, \mathrm{D}-/ \mathrm{R}+5$, and $\mathrm{D}-/ \mathrm{R}-4$. Valganciclovir was given for a total of 6 months posttransplant except for D-/R+ patients who received it for 12 months. Results. Five patients (12\%) developed CMV disease with an average followup of 26 months. Only 2 (4.7\%) developed CMV disease within six months of completing valganciclovir prophylaxis. This incidence is not significantly different from the best-reported results of CMV prophylaxis in lung transplant recipients. The remaining 3 patients developed the disease later in their course, one as late as 32 months posttransplant. The main side effects noted include leucopenia, neutropenia, and GI disturbances. However, the number of patients who had to temporarily stop or discontinue the medication (9.5\%) was significantly lower than that reported in previous studies. Conclusions. Our experience suggests that low-dose valganciclovir is an effective method of prophylaxis for CMV disease in high-risk patients. It is a simple regimen that seems to have a better side effect profile and to improve patient compliance.
\end{abstract}

\section{Introduction}

Cytomegalovirus (CMV) disease is the leading cause of opportunistic infection in immunocompromised subjects following solid organ transplantation (SOT) [1]. The rate of $\mathrm{CMV}$ infection and disease in lung transplant recipients is higher than other solid organ transplant groups [2]. Lung and heart-lung transplant recipients who are not receiving antiviral prophylaxis have a reported incidence of CMV disease ranging from $38 \%$ to $75 \%$ [3]. Besides causing pneumonitis and direct invasion of other organ tissues such as the gastrointestinal tract, CMV has immunomodulatory effects. As a result, CMV disease is an independent risk factor for development of other bacterial and fungal infections as well as other viral infections such as Epstein-Barr virus and its related posttransplant lymphoproliferative disease $[4,5]$. In addition, CMV may be an important risk factor for the development of bronchiolitis obliterans syndrome 
(BOS) after lung transplantation, which is the major cause of mortality and morbidity in lung transplant recipients beyond one year of transplant [6,7]. Given the potential adverse complications and high risk of developing CMV disease, universal antiviral prophylaxis against CMV is adopted by most lung transplant centers.

Prevention of CMV infection and viremia has been well documented in lung transplant recipients using primary or secondary prophylaxis methods as well as avoiding the use of blood products from CMV positive donors and matching lung transplant recipients with donors that are CMV negative. Different modes of drug prophylaxis have been studied in this patient population, which include a combination or individual use of oral and intravenous ganciclovir, oral valganciclovir, and CMV immune globulins. Using these forms of prophylaxis, the incidence of CMV disease after lung transplantation has been shown to decrease to about 10-20\% $[8,9]$.

There is no consensus on the optimal form and duration of CMV prophylaxis. Both the oral and intravenous forms of ganciclovir have been shown in the single organ transplant (SOT) population to be equally effective for CMV prophylaxis $[10,11]$. The limitations of oral ganciclovir (1 gm TID) are mainly due to poor oral absorption and low bioavailability, requiring a thrice daily dosing regimen. Intravenous ganciclovir $(5 \mathrm{mg} / \mathrm{kg})$ is limited by its associated complications of catheter related infections and thrombosis. This led to the development of valganciclovir (Valcyte). Valganciclovir is an oral prodrug of ganciclovir that has significantly enhanced oral bioavailability compared with oral ganciclovir $(60.9 \%$ versus 5.6\%) allowing for a convenient once or twice daily dosing regimen [12]. This, along with favorable dosing, has led most centers to use the better tolerated valganciclovir as the preferred CMV prophylaxis.

Most recent studies done for CMV prophylaxis in lung transplant recipients have used high-dose valganciclovir (900 mg-1800 mg daily). In a clinical trial that evaluated the efficacy of valganciclovir prophylaxis in SOT patients, leucopenia and neutropenia were observed to be more common in patients who received high-dose valganciclovir as compared to those who received standard doses of oral ganciclovir [13]. This may be related to higher systemic exposure to ganciclovir after valganciclovir administration, since a nonsignificant trend was observed towards a higher incidence of neutropenia and leucopenia with higher systemic exposures to ganciclovir [14]. Systemic exposure to ganciclovir using Low-Dose valganciclovir at $450 \mathrm{mg}$ daily is equivalent to $3 \mathrm{gm}$ daily of ganciclovir [15]. $3 \mathrm{gm}$ of oral ganciclovir has been shown to be equally effective to highdose valganciclovir (900 mg) for CMV prevention in high risk SOT recipients [13]. Therefore, the lower dose of $450 \mathrm{mg}$ of valganciclovir would be expected to be equivalent in efficacy to the higher doses, but with fewer side effects. However, this Low-Dose of valganciclovir has never been critically assessed in lung transplant patients.

We present our experience at a single center, in a retrospective chart analysis of 42 consecutive lung transplant recipients who were given $450 \mathrm{mg}$ daily of oral valganciclovir for CMV prophylaxis. The drug was continued for 6 or 12 months, depending upon the donor (D) and recipients (R) $\mathrm{CMV}$ status. Our hypothesis is that oral valganciclovir at a lower dose of $450 \mathrm{mg}$ daily is equally effective for CMV prophylaxis in lung transplant recipients as the currently recommended dose of $900 \mathrm{mg}$ once or twice a day, and that at this lower dosage, it will have a better side effect profile leading to fewer complications and better patient compliance.

\section{Patients and Methods}

The study was conducted at the lung transplant center at Cedar Sinai Medical Center and there was a review of records with waiver of informed consents for reviewing that was approved by the institutional review board.

2.1. Study Design. This is a retrospective chart analysis using electronic medical records. All patients who received lung transplant from Jan 2001 to Dec 2006 at our center were reviewed separately by two independent clinical investigators. Out of a total of 46 patients who received lung transplant at our center, 4 were excluded from our study because they died within thirty days of surgery from postoperative complications. The electronic medical records were reviewed to evaluate multiple variables including the reason of transplantation, single or double lung transplantation, age at transplantation, incidence of acute rejection, CMV status of the donor and recipient, incidence of CMV disease, time of onset of CMV disease from transplantation, incidence of leukopenia, and anemia and GI intolerance while on valganciclovir along with incidence of discontinuation of valganciclovir due to any of these side effects.

All patients except for the low-risk (Donor (D)-/Recipient $(\mathrm{R})-$ ) patients received primary prophylaxis for CMV disease with $450 \mathrm{mg}$ daily of valganciclovir given orally starting as soon as they were able to take oral medication. Prophylaxis was continued for at least 6 months except for highrisk $(\mathrm{D}+/ \mathrm{R}-)$ patients who received oral valganciclovir for a total of 12 months posttransplant. Dose adjustments were not made for a decrease in GFR during the study period, although subsequently our practice has evolved to make adjustments in valganciclovir dosing for GFR. All patients received induction immunosuppression at the time of transplantation with daclizumab $1 \mathrm{mg} / \mathrm{kg}$ followed by triple drug immunosuppressive therapy with a calcineurin inhibitor, mycophenolic acid, and prednisone started immediately following the transplant procedure. Calcineurin levels were checked, and doses were adjusted to maintain therapeutic levels between 10 and $20 \mathrm{mg} / \mathrm{dL}$ for tacrolimus and between 200 and $300 \mathrm{mg} / \mathrm{dL}$ for cyclosporine. Mycophenolic acid was started at a dose of $1 \mathrm{gm}$ orally twice a day and then adjusted to maintain a total WBC count between 4.0 and 10.0. Prednisone was dosed between 0.2 and $0.3 \mathrm{mg} / \mathrm{kg}$ daily and continued indefinitely. All patients were also placed on pneumocystis prophylaxis with bactrim. All transplant patients were followed closely in the posttransplant clinic, weekly for the first month, every other week for the next 2 months, monthly for the next 6 months, and finally every 3 months. CMV PCR and cultures were obtained when patients developed any clinical signs 
and symptoms of CMV disease. Cultures were not routinely drawn to look for asymptomatic viremia nor were CMV antigenemia assays were routinely done. Bronchoscopies with transbronchial biopsies were performed with any clinical sign or symptom of infection, but no surveillance biopsies were done.

2.2. Definitions. CMV disease was defined as evidence of tissue invasion based on transbronchial biopsy or a positive tissue culture or a syndrome with fever, malaise, leucopenia or diarrhea, or hepatic transaminitis with a positive CMV PCR. Leukopenia was defined by a white blood cell count less than 3,500, neutropenia as absolute neutrophil count less than 1,500, anemia as a hemoglobin less than 12, and thrombocytopenia as platelet count less than 100,000.

2.3. Treatment of $C M V$ Disease. For patients diagnosed with CMV disease, a 3-week course of oral valganciclovir $900 \mathrm{mg}$ twice a day was completed [16] as tolerated and was then followed by secondary prophylaxis with oral valganciclovir at $450 \mathrm{mg}$ daily for at least an additional six months. For patients with severe CMV disease, intravenous ganciclovir therapy was initiated and transitioned to oral valganciclovir when clinically improved and able to take oral medication. CMV specific immune globulin (Cytogam) was also used on a case by case basis in severe cases of CMV disease.

\section{Results}

A total of 46 patients' electronic medical records were reviewed. 4 patients were excluded because they died within 30 days of the transplant from noninfectious causes. 42 patients were included in this study. Demographic and clinical characteristics are presented in Table 1. A total of 5 out of $42(12 \%)$ patients developed CMV disease after lung transplant. These as well as all the other patients who did not develop CMV disease had a negative viral load as determined by CMV-PCR before discontinuing prophylaxis. Mean followup was 26 months posttransplant. Only 2 out of $42(4.7 \%)$ patients developed CMV disease within 6 months of completing oral valganciclovir prophylaxis. None of the patients who developed CMV disease were on oral valganciclovir at the time of onset of the disease. There was one death attributed to CMV disease complicated by respiratory failure and bronchial anastomotic stricture (Tables 2 and 3 ).

Leukopenia occurred in 19 out of 42 patients (42\%), and anemia occurred in 30 out of the 42 (71\%) while on oral valganciclovir prophylaxis, four out of the 42 patients $(9.5 \%)$ had their valganciclovir stopped at least temporarily due to leucopenia. Eight out of 42 patients had significant complaints of gastrointestinal intolerance during their posttransplant course, which did not require discontinuation valganciclovir prophylaxis. Fourteen out of 42 patients (33\%) developed opportunistic infections other than CMV (mainly Aspergillus, Mucor, and Cryptococcus infections) during the posttransplant course, and 11 out of the 42 had evidence of acute rejection during the after lung transplant course.
TABLE 1: Baseline demographic data for the Valganciclovir study group.

\begin{tabular}{lc}
\hline Age, mean (years) & 64 \\
Gender: male/female & $25 / 17$ \\
Indication for transplant, $n$ (\%) & \\
$\quad$ Chronic obstructive pulmonary disease & $26(62 \%)$ \\
Interstitial lung disease & $4(10 \%)$ \\
Idiopathic pulmonary fibrosis & $6(14 \%)$ \\
Cystic fibrosis & $3(7 \%)$ \\
Systemic sclerosis with pulmonary hypertension & $1(2 \%)$ \\
Alpha 1-antitrypsin deficiency & $2(4 \%)$ \\
CMV donor/recipient serostatus, $n(\%)$ & \\
D+/R+ & $28(67 \%)$ \\
D+/R- & $5(12 \%)$ \\
D-/R+ & $5(12 \%)$ \\
D-/R- & $4(9 \%)$ \\
Single/bilateral lung transplant & $36 / 6$ \\
Acute rejection episodes & $11(26 \%)$ \\
Evidence of opportunistic infections & \\
Aspergillus & $12(28 \%)$ \\
Mucor & $2(5 \%)$ \\
Cryptococcus & $2(5 \%)$ \\
\hline
\end{tabular}

\section{Discussion}

Cytomegalovirus (CMV) remains an important pathogen following solid organ transplantation (SOT) and a significant cause of mortality and morbidity in this patient population. Infection with CMV can present with an acute viral syndrome associated with fever and other constitutional symptoms as well as various specific end-organ syndromes including pneumonitis, enterocolitis, nephritis, and hepatitis. CMV may also have indirect (secondary) effects, including allograft injury, rejection, and increased risk of other opportunistic infection. Due to the presence of a large amount of lymphoid tissue in the lung, transplantation of this vital organ involves the transfer of a high viral load of latent donor CMV virus compared to other solid organs transplants [2]. Consequently the risk of developing CMV infection and disease may increase in this patient population with a reported incidence as high as $75 \%[3]$.

There are several options currently available for CMV prophylaxis including intravenous and oral ganciclovir, oral valganciclovir, and CMV hyperimmune globulin. Ganciclovir is effective prophylaxis for CMV disease, but major limitation of oral ganciclovir includes poor oral absorption [10]. Intravenous ganciclovir with its associated complications of catheter-related infusions and thrombosis is impractical. Valganciclovir is an oral prodrug of ganciclovir and has significantly enhanced oral bioavailability compared with oral ganciclovir (60.9\% versus 5.6\%) allowing for a convenient once or twice daily dosing regimen [12]. Systemic exposure to ganciclovir after $450 \mathrm{mg}$ of oral valganciclovir given once daily was comparable to that of oral ganciclovir, 
TABLE 2: Characteristics of the people who developed CMV disease.

\begin{tabular}{lccc}
\hline Patient's age at transplant & CMV serostatus D/R & $\begin{array}{c}\text { Time after transplant to } \\
\text { develop CMV disease }\end{array}$ & $\begin{array}{c}\text { Time after stopping valganciclovir } \\
\text { to develop CMV disease }\end{array}$ \\
\hline 52 years & D+/R- & 32 months & 24 months \\
66 years & $\mathrm{D}+/ \mathrm{R}+$ & 8 months & 2 months \\
60 years & $\mathrm{D}-/ \mathrm{R}-$ & 23 months & 17 months \\
57 years & $\mathrm{D}+/ \mathrm{R}-$ & 15 months & 9 months \\
70 years & $\mathrm{D}+/ \mathrm{R}+$ & 8 months & 5 months \\
\hline
\end{tabular}

TABLE 3: Zamora study group demographic data.

\begin{tabular}{lc}
\hline Age, mean & $53.4 \pm 4.7$ \\
Gender: male/female & $50 / 40$ \\
Indications for transplant, $n(\%)$ & \\
$\quad$ COPD & $45(50)$ \\
Alpha 1-antitrypsin deficiency & $21(23.3)$ \\
Idiopathic pulmonary fibrosis & $8(8.9)$ \\
Cystic fibrosis & $12(13.3)$ \\
Primary pulmonary hypertension & $4(5.5)$ \\
CMV donor/recipient serostatus, $n(\%)$ & \\
D+/R- & $18(20)$ \\
D+/R+ & $45(50)$ \\
D-/R+ & $27(30)$ \\
\hline
\end{tabular}

at a dose of $1 \mathrm{gm}$ three times daily. The systemic exposure to ganciclovir after $900 \mathrm{mg}$ of oral valganciclovir given once daily was comparable to intravenous ganciclovir, at a dose of $5 \mathrm{mg} / \mathrm{kg}$ daily $[15,17]$. In a heterogeneous cohort of solid organ transplant patients, the daily systemic exposure (area under the curve in $24 \mathrm{hrs}$ of drug exposure) to ganciclovir was 1.7 times higher after valganciclovir at $900 \mathrm{mg}$ once a day than after oral ganciclovir administration at $1000 \mathrm{mg}$ three times a day [17]. This was demonstrated more recently in a small cohort of lung transplant recipients as well, where $900 \mathrm{mg}$ daily of valganciclovir resulted in the equivalent of a mean daily dose of $7.7 \mathrm{mg} / \mathrm{kg}$ intravenous ganciclovir [18].

The optimal duration of CMV prophylaxis is unknown, but in general, the longer the duration of prophylaxis, the lower the incidence of primary or recurrent CMV disease. This has been demonstrated in kidney transplant recipients after six months of CMV prophylaxis with valganciclovir [19], and in recent studies by Humar and Zamora et al. $[20,21]$, it has been demonstrated that a longer duration of prophylaxis is beneficial. However, it is not clear how much exposure to ganciclovir is required for effective prophylaxis. In a randomized trial of daily versus thrice-weekly prophylactic ganciclovir after lung and heart-lung transplantation, there was no significant difference in the incidence of CMV infection or disease [22]. Valganciclovir at a dosage of $900 \mathrm{mg}$ daily was compared with oral ganciclovir at $1000 \mathrm{mg}$ three times a day (which leads to similar ganciclovir exposure as with valganciclovir $450 \mathrm{mg}$ daily) in a large multicenter trial of $364 \mathrm{CMV} \mathrm{D+/R-} \mathrm{solid} \mathrm{organ} \mathrm{transplant} \mathrm{recipients}$ [13]. This trial demonstrated comparable efficacy of the two regimens as well as good viral suppression while on therapy. Again, viremia and disease were most commonly seen after the discontinuation of prophylaxis. Extended duration of ganciclovir exposure seems to have a better prophylactic effect than a short course, and the higher dosage does not seem to have a beneficial effect but tends to cause more significant side effects including neutropenia and gastrointestinal intolerance leading to higher discontinuation rates and poor patient compliance. In addition, the ease and convenience of a once daily dosing regimen are likely to improve compliance and quality of life amongst patients who are already taking large amount of other medications following transplantation.

In a clinical trial that evaluated the efficacy of valganciclovir prophylaxis in solid organ transplant patients, leucopenia and neutropenia were observed to be more common in patients who received high-dose valganciclovir as compared to those who received standard doses of oral ganciclovir [13]. This may be related to higher systemic exposure to ganciclovir after valganciclovir administration, since a nonsignificant trend was observed towards a higher incidence of neutropenia and leucopenia with higher systemic exposures to ganciclovir [14]. Other than leucopenia and neutropenia, the safety profile of $900 \mathrm{mg}$ once daily of valganciclovir was similar to $1000 \mathrm{mg}$ three times daily of oral ganciclovir. However, these side effects tend to reduce the duration of CMV prophylaxis that lung transplant recipients tolerate thus increasing the risk of CMV disease with shorter prophylaxis regimens [23].

In this study, we present our single center experience using $450 \mathrm{mg}$ daily of oral valganciclovir as universal CMV prophylaxis after lung transplantation. Depending upon the CMV serostatus, valganciclovir was given for 6 months or 12 months following lung transplantation. Low-risk patients who had negative donor and negative recipient CMV serostatus did not receive valganciclovir prophylaxis and were not included in the analysis. We report in our retrospective analysis an incidence of CMV disease of $12 \%$ with a mean followup of 26 months posttransplant. Prior to initiation of this protocol, the incidence of CMV disease at our center using IV ganciclovir prophylaxis was similar. Most of the published studies on CMV prophylaxis in lung transplant recipients have had an average followup of 6 to 12 months only. These studies may have missed delayed cases of CMV disease following discontinuation of prophylaxis. Since there is lack of a large randomized trial for optimum prophylaxis for CMV disease following lung transplantation, there is still no consensus on the best approach. Different centers have 
varying dosages, drugs, and regimens for CMV prophylaxis, which makes comparison between different reported results difficult with some using IV ganciclovir, CMV immune globulins, and valganciclovir in different combinations and varying lengths of time. Recently Zamora et al. [21] evaluated valganciclovir for CMV prophylaxis in lung transplant recipients. All patients received 30 days of IV ganciclovir and three doses of CMV immune globulin (if $\mathrm{R}+$ ) or 90 days of IV ganciclovir with seven doses of CMV immune globulin (if $\mathrm{D}+\mathrm{R}-$ ). This was followed by oral valganciclovir of $900 \mathrm{mg}$ a day for attempted durations of 180, 270, and 365 days. They compared the incidence of CMV disease in these patients with a historical control and showed a significant improvement (2.2\% versus $20 \%)$. Patients were followed for a total of 6 months after stopping oral valganciclovir. This is the best-reported incidence of CMV disease in the literature. With a similar followup time, there is no significant difference in our incidence of CMV disease within 6 months of discontinuation of valganciclovir prophylaxis at the lower dose $(4.7 \%$ versus $2.2 \%$ with a $P$ value of 0.8$)$. However, the number of patients who had to discontinue valganciclovir due to side effects was significantly lower in our patient population $(9.5 \%$ versus $32 \%$ with a $P$ value of 0.01$)$. Thus, the incidence of CMV disease in our lung transplant patients is similar to the best reported in the literature to date, but our prophylactic regimen seems to be better tolerated, with fewer significant side effects leading to better patient compliance. This data, however, needs to be interpreted in light of the fact that our patient populations are different and that our immune-suppression regimens are not identical.

CMV resistance to ganciclovir has become a growing concern; however, the clinical relevance of this is still unknown [24]. It is likely the result of both the magnitude and duration of antiviral exposure to systemic ganciclovir. There are some concerns that at a Low-Dose valganciclovir may promote development of ganciclovir resistance. However, there is no data to substantiate these concerns. We did not monitor ganciclovir resistance in our cohort, but all the patients who developed CMV disease responded well to oral valganciclovir at a higher dosage or intravenous ganciclovir. Also our data supports the current understanding that anti CMV prophylaxis does not completely eliminate the risk of developing CMV disease but often simply delays the onset of disease. This changing epidemiology of CMV disease following solid organ transplantation is now a well-recognized consequence of the widespread use of antiviral prophylaxis. With our longer duration of followup, we now have reported cases of CMV disease developing as late as three years posttransplant. Whether this suggests that a more prolonged course of prophylaxis is beneficial will require further study. We believe that the patients in this study who developed CMV disease after discontinuation of oral valganciclovir did so due to "early" discontinuation of prophylaxis rather than insufficient dosing during prophylaxis and could have benefited from longer duration of prophylaxis.

Our paper has some obvious limitations. Clearly a prospective randomized clinical trial would be a superior study design. The optimal regimen for CMV prophylaxis in solid organ transplant patients needs further evaluation with a randomized trial, taking into account the changing epidemiology of the disease, the availability of simpler dosing regimens, the clinical significance of asymptomatic viremia, and the emergence of viral resistance.

In summary, we report our experience with Low-Dose of oral valganciclovir for a longer duration of time which seems to be safe and effective for CMV prophylaxis with much lower incidence of side effects and complications leading to better patient compliance and reduced rate of discontinuation of the drug.

\section{References}

[1] J. A. Fishman and R. H. Rubin, "Infection in organ-transplant recipients," The New England Journal of Medicine, vol. 338, no. 24, pp. 1741-1751, 1998.

[2] M. Balthesen, M. Messerle, and M. J. Reddehase, "Lungs are a major organ site of cytomegalovirus latency and recurrence," Journal of Virology, vol. 67, no. 9, pp. 5360-5366, 1993.

[3] B. D. Alexander and V. F. Tapson, "Infectious complications of lung transplantation," Transplant Infectious Disease, vol. 3, no. 3, pp. 128-137, 2001.

[4] D. R. Snydman, "Infection in solid organ transplantation," Transplant Infectious Disease, vol. 1, no. 1, pp. 21-28, 1999.

[5] N. Singh, P. M. Arnow, A. Bonham et al., "Invasive aspergillosis in liver transplant recipients in the 1990s," Transplantation, vol. 64, no. 5, pp. 716-720, 1997.

[6] S. Husain and N. Singh, "Bronchiolitis obliterans and lung transplantation: evidence for an infectious etiology," Seminars in Respiratory Infections, vol. 17, no. 4, pp. 310-314, 2002.

[7] J. Cerrina, F. Le Roy Ladurie, P. H. Herve et al., "Role of CMV pneumonia in the development of obliterative bronchiolitis in heart-lung and double-lung transplant recipients," Transplant International, vol. 5, supplement 1, pp. S242-S245, 1992.

[8] D. Weill, B. J. Lock, D. L. Wewers et al., "Combination prophylaxis with ganciclovir and cytomegalovirus (CMV) immune globulin after lung transplantation: effective CMV prevention following daclizumab induction," American Journal of Transplantation, vol. 3, no. 4, pp. 492-496, 2003.

[9] M. R. Zamora, "Use of cytomegalovirus immune globulin and ganciclovir for the prevention of cytomegalovirus disease in lung transplantation," Transplant Infectious Disease, vol. 3, no. 2, pp. 49-56, 2001.

[10] E. Gane, F. Saliba, G. J. C. Valdecasas et al., "Randomised trial of efficacy and safety of oral ganciclovir in the prevention of cytomegalovirus disease in liver-transplant recipients," The Lancet, vol. 350, no. 9093, pp. 1729-1733, 1997.

[11] S. R. Duncan, I. L. Paradis, J. H. Dauber, S. A. Yousem, R. L. Hardesty, and B. P. Griffith, "Ganciclovir prophylaxis for cytomegalovirus infections in pulmonary allograft recipients," American Review of Respiratory Disease, vol. 146, no. 5, pp. 1213-1215, 1992.

[12] M. D. Pescovitz, J. Rabkin, R. M. Merion et al., "Valganciclovir results in improved oral absorption of ganciclovir in liver transplant recipients," Antimicrobial Agents and Chemotherapy, vol. 44, no. 10, pp. 2811-2815, 2000.

[13] C. Paya, A. Humar, E. Dominguez et al., "Efficacy and safety of valganciclovir vs. oral ganciclovir for prevention of cytomegalovirus disease in solid organ transplant recipients," American Journal of Transplantation, vol. 4, no. 4, pp. 611-620, 2004. 
[14] H. Wiltshire, S. Hirankarn, C. Farrell, K. Zuideveld, and PV, 16000 Solid Organ Transplantation Study Group, "Cytomegalovirus suppression in relation to levels of ganciclovir exposure following valganciclovir prophylaxis in solid organ transplant recipients," in Proceedings of the 43rd Interscience Conference on Antimicrob. Agents Chemother, vol. 40, American Society for Microbiology Press, Chicago, Ill, USA, 2003.

[15] M. D. Pescovitz, J. Rabkin, R. M. Merion et al., "Valganciclovir results in improved oral absorption of ganciclovir in liver transplant recipients," Antimicrobial Agents and Chemotherapy, vol. 44, no. 10, pp. 2811-2815, 2000.

[16] L. S. Munoz, M. Angelis et al., "Valganciclovir for the treatment of CMV infections in transplant patients," Infectious Disease Society of America, vol. 98, abstract 366, 2003.

[17] M. D. Pescovitz, "Oral ganciclovir and pharmacokinetics of valganciclovir in liver transplant recipients," Transplant Infectious Disease, vol. 1, pp. 31-34, 1999.

[18] T. H. Kiser, D. N. Fish, and M. R. Zamora, "Evaluation of valganciclovir pharmacokinetics in lung transplant recipients," The Journal of Heart and Lung Transplantation, vol. 31, no. 2, pp. 159-166, 2011.

[19] I. Helanterä, L. Kyllönen, I. Lautenschlager, K. Salmela, and P. Koskinen, "Primary CMV infections are common in kidney transplant recipients after 6 months valganciclovir prophylaxis," American Journal of Transplantation, vol. 10, no. 9, pp. 2026-2032, 2010.

[20] A. Humar, D. Kumar, J. Preiksaitis et al., "A trial of valganciclovir prophylaxis for cytomegalovirus prevention in lung transplant recipients," American Journal of Transplantation, vol. 5, no. 6, pp. 1462-1468, 2005.

[21] M. R. Zamora, M. R. Nicolls, T. N. Hodges et al., "Following universal prophylaxis with intravenous ganciclovir and cytomegalovirus immune globulin, valganciclovir is safe and effective for prevention of CMV infection following lung transplantation," American Journal of Transplantation, vol. 4, no. 10, pp. 1635-1642, 2004.

[22] M. I. Hertz, C. Jordan, S. K. Savik et al., "Randomized trial of daily versus three-times-weekly prophylactic ganciclovir after lung and heart-lung transplantation," Journal of Heart and Lung Transplantation, vol. 17, no. 9, pp. 913-920, 1998.

[23] V. Monforte, C. Lopez, F. Santos et al., "A multicenter study of valganciclovir prophylaxis up to day 120 in CMV-Seropositive lung transplant recipients," American Journal of Transplantation, vol. 9, no. 5, pp. 1134-1141, 2009.

[24] E. Akalin, V. Sehgal, S. Ames et al., "Cytomegalovirus disease in high-risk transplant recipients despite ganciclovir or valganciclovir prophylaxis," American Journal of Transplantation, vol. 3, no. 6, pp. 731-735, 2003. 


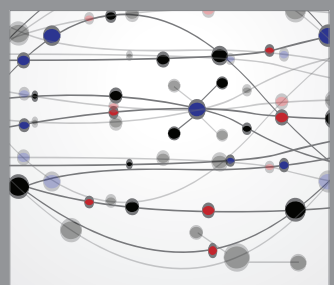

The Scientific World Journal
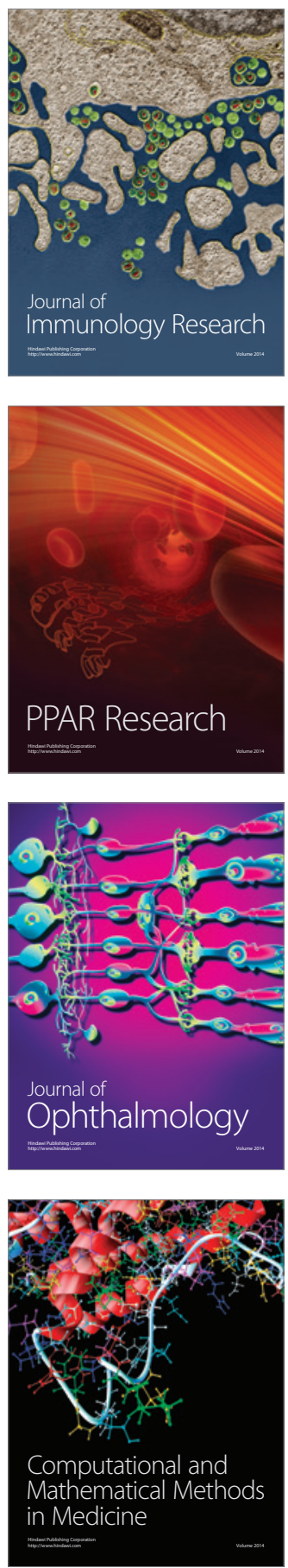

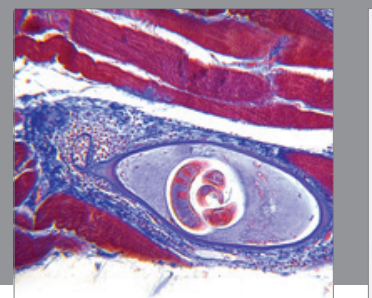

Gastroenterology

Research and Practice
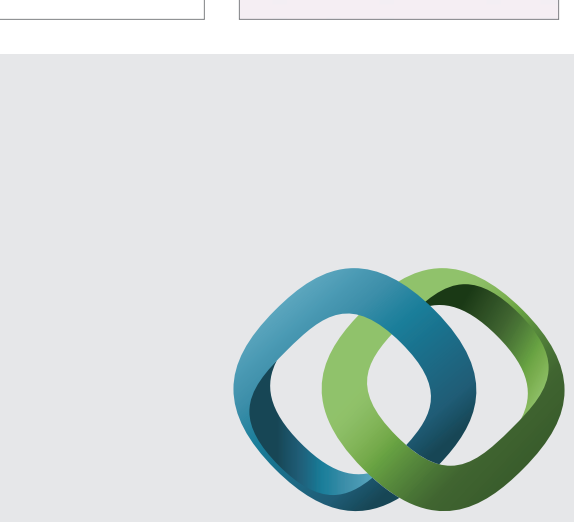

\section{Hindawi}

Submit your manuscripts at

http://www.hindawi.com
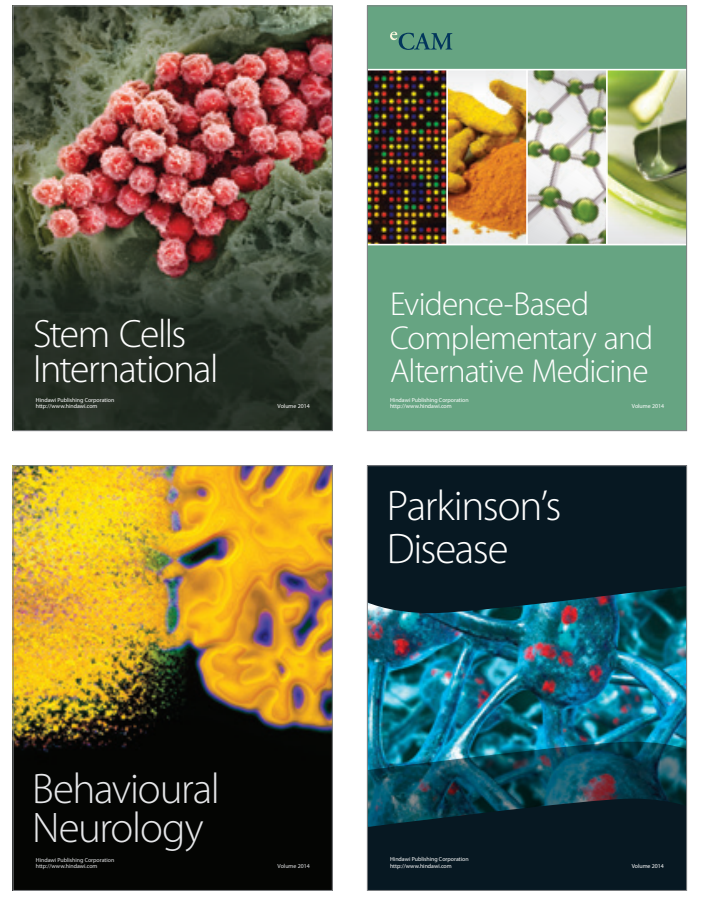
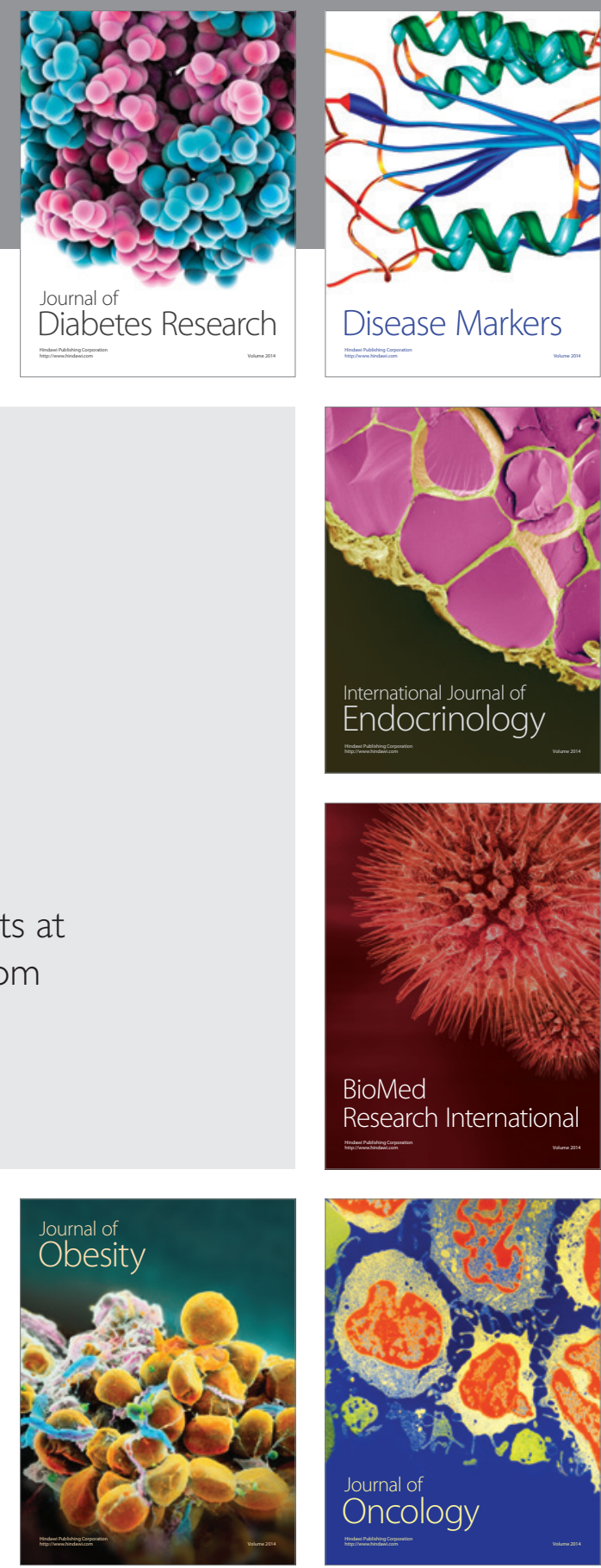

Disease Markers
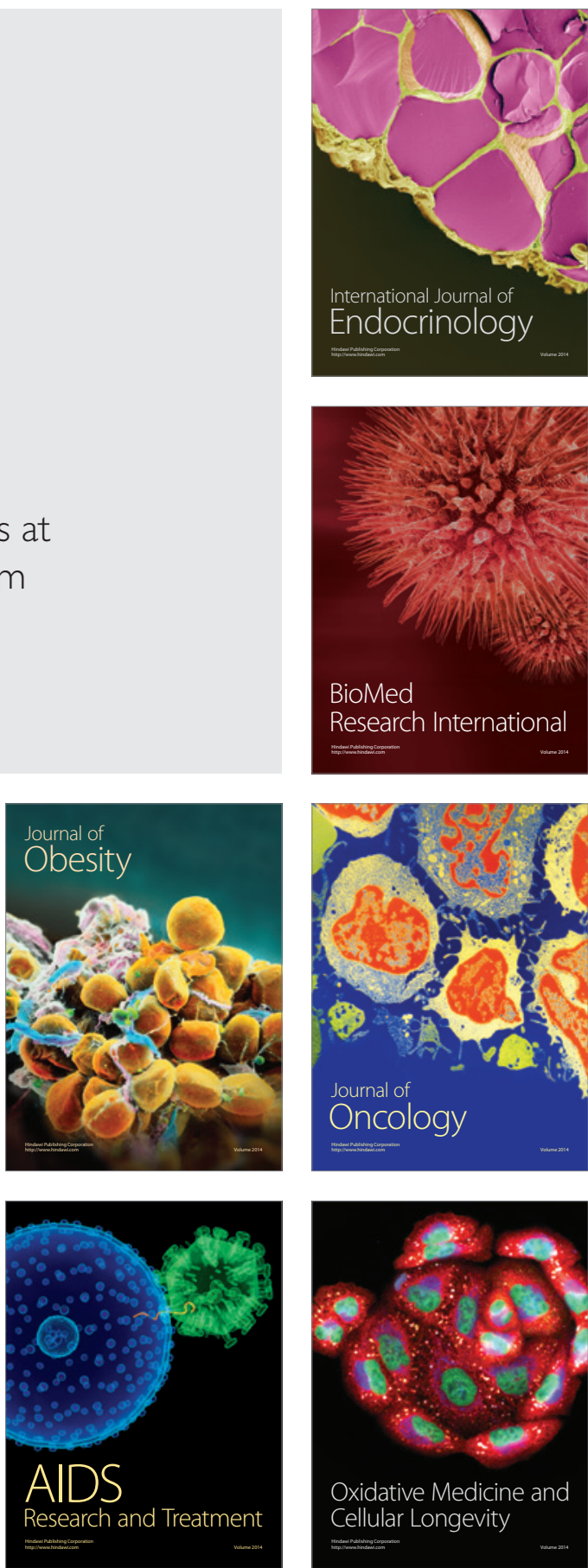